[0212-7199 (2007) 24: 7; pp 317-323] ANALES DE MEDICINA INTERNA Copyright (C) 2007 ARAN EDICIONES, S.L.

AN. Med. InTERna (Madrid) Vol. 24, N. ${ }^{\circ}$, pp. $317-323,2007$

\section{Análisis transversal de la insuficiencia cardiaca en pacientes de un servicio de medicina interna de un hospital de tercer nivel de área mixta (rural y urbana). Parte II: prevalencia y grado de control de hipertensión arterial (Segunda de tres partes)}

\author{
S. CINZA SANJURJO, A. CABARCOS ORTIZ DE BARRÓN' ${ }^{1}$ E. NIETO POL ${ }^{2}$, \\ J. A. TORRE CARBALLADA ${ }^{1}$
Fundación Pública Urgencias Sanitarias 061. 'Servicio de Medicina Interna. Complejo Hospitalario Universitario. ${ }^{2}$ Servicio de Atención Primaria. Centro de Salud Concepción Arenal. Santiago de Compostela. A Coruña

CROSS-SECTIONAL STUDY OF HEART FAILURE OF PATIENTS INTAKED IN AN INTERNAL MEDICINE SERVICE IN THE THIRD LEVEL HOSPITAL IN MIXED AREA. PART II: PREVALENCE AND HYPERTENSION CONTROL

\section{RESUMEN}

Objetivos: Conocer la prevalencia y grado de control de la hipertensión arterial entre los pacientes con insuficiencia cardiaca.

Material y Métodos: Estudio descriptivo transversal, retrospectivo, de pacientes ingresados en el Servicio de Medicina Interna del Hospital Clínico Universitario de Santiago de Compostela a lo largo de 5 años (desde 1999 hasta 2003). Se registraron las variables: sexo, edad, días de ingreso, número de reingresos por insuficiencia cardíaca, motivo de ingreso, hipertensión arterial (HTA) previa, diabetes mellitus (DM) previa, cardiopatía previa, cardiopatía isquémica previa, fibrilación auricular (FA) previa, consumo de beta-bloqueantes previos al ingreso, cifras de presión arterial (PA) al ingreso, realización de ecocardiografía durante el ingreso, fracción de eyección (FE) según ecocardiograma, factor desencadenante, exitus, tratamiento domiciliario al alta. Para el análisis estadístico se emplearon los estadísticos descriptivos cuantitativos y cualitativos que correspondieran; para el análisis bivariante se emplearon chi-cuadrado y " $t$-student".

Resultados: Se incluyeron un total de 248, de los cuales $100(41,8 \%)$ eran hipertensos. Se observó un predominio de mujeres en el grupo de hipertensos $(63,0 \%)$, similar a los no hipertensos $(51,1 \%), \mathrm{p}=0,06$. La mediana de edad entre hipertensos fue de 77 años (rango intercuartílico de 10 años) similar a los no hipertensos (mediana de 77 años y rango intercuartílico de 14 años), $p=0,64$. La mediana de tiempo de ingreso fue de 11 días entre hipertensos (10 días, rango intercuartílico 8,5 días) y no hipertensos (11 días, rango intercuartílico 9 días). El análisis de los antecedentes personales mostró que el número de diabéticos y de pacientes con cardiopatía isquémica era claramente superior en el grupo de hipertensos $(43,0$ vs. $22,3 \%), \mathrm{p}<0,001 ;(38,0$ vs. $21,6 \%), \mathrm{p}=0,005$; respectivamente. El síntoma que originó el ingreso con más frecuencia fue la disnea en hipertensos $(73,0 \%)$ y no hipertensos $(66,9 \%), p=0,62$. En el momento del ingreso el $62,6 \%$ de la población estudiada presentaba cifras anormalmente elevadas de presión arterial (PA). En el caso de los hipertensos, esta prevalencia alcanza el $76,9 \%$, permaneciendo en $59,4 \%$ en no hipertensos, $p=0,01$. La media de presión arterial sistólica (PAS) y presión arterial diastólica (PAD) fue superior en pacientes hipertensos, $\mathrm{p}<$ 0,01 en ambos casos. El número de ecocardiogramas realizados entre hipertensos y no hipertensos fue similar $(16,3$ vs. $24,8 \%), p=0,12$. Los pacientes con disfunción diastólica fue ligeramente superior en hipertensos, sin ser una diferencia estadísticamente significativa $(62,5 v s .50,5 \%)$, $\mathrm{p}=0,41$.

Entre los tratamientos administrados se observó que se administraban más diuréticos en el grupo de pacientes no hipertensos $(91,1 \%$ vs $81,1 \%, \mathrm{p}=0,03)$. El grupo IECA/ARA-II fue significativamente más administrado en el grupo de hipertensos $(62,6 \%$ vs $26,8 \%$, $\mathrm{p}<0,001)$.

\section{ABSTRACT}

Objectives: To know the arterial hypertension prevalencia and hypertension control in the patients income by heart failure.

Methods: A cross-sectional study of the intaked patients in the Internal Medicine Service in the Hospital Clínico Universitario de Santiago de Compostela between 1999 to 2003. The variables analized were: sex, for admission (guide symptom), hypertension, diabetes mellitus, cardiac disease, fibrillation atrium, previous treatment with beta-blockers, blood pressure in the admission moment, to make echocardiography, disfunction systolic, etiology, deceased, treatment at the end. The statistical analysis was performed with cualitative and cuantitative measures, chicuadrado and t-student, and multivariant analyses.

Results: 248 patients were accepted for the study, and 100 were hypertensive patients (41.8\%). We observed more women than men in hypertensive group (63.0\%) and in non hypertensive group (51.1\%). The median age was 77 years old in both groups. The median income was 11 days. The number of patients with diabetes mellitus and ischemic cardiopathy was bigger in hypertension group (43.0 vs. 22.3\%), $p<0.001$; (38vs. 21.6\%), $p=0.005$. The most frequent simptom was the dyspnea trol of blood pressures. The prevalence of bad control in hypertensive patients was bigger tha non-hypertensive patients (76.9 vs. 59.4\%, $p=$ $0.01)$. The pharmacologic treatment more prescribed in hypertensive patients ECAI or AAR-II (62.6 vs. 26.8\%, $p<0.001)$. And the diuretics wee more prescribed in non-hypertensive patients (91.1 vs. $81.1 \%, p=0.03)$.

Conclusions: The prevalence of diabetes mellitus is associated with hypertension in the patients. The ECAI prescription was acceptable. The number of echocardiograms practiced to the patients is smaller that the number advised by international associations and smaller to the cardiologist registers. The beta-blockers is smaller too. age, days of hospital stay, number of intaked by failure cardiac, reason $(66,9 \%)$, in both groups, $p=0.62$. The $62.6 \%$ of the patients were bad con- 
Conclusiones: Se observa una elevada comorbilidad entre diabetes mellitus e hipertensión. La realización de ecocardiogramas es muy baja en pacientes hipertensos y con insuficiencia cardiaca. El empleo de IECAS es consistente con los protocolos actuales. La prescripción de beta-bloqueantes sigue siendo muy baja respecto a las recomendaciones de las sociedades internacionales.

PALABRAS CLAVE: Insuficiencia cardíaca. Epidemiología. Ecocardiogramas. Hipertensión Arterial.
KEY WORDS: Heart failure. Epidemiology. Echocardiogram. Hypertension.

Cinza Sanjurjo S, Cabarcos Ortiz de Barrón A, Nieto Pol E, Torre Carballada JA. Análisis transversal de la insuficiencia cardiaca en pacientes de un servicio de medicina interna de un hospital de tercer nivel de área mixta (rural y urbana). Parte II: prevalencia y grado de control de hipertensión arterial. An Med Interna (Madrid) 2007; 24: 317-323.

\section{INTRODUCCIÓN}

La insuficiencia cardiaca representa una de las patologías más prevalentes en los países desarrollados (1), alcanzando una prevalencia del $10 \%$ en mayores de 70 años (2), esto se debe fundamentalmente al envejecimiento de la población y a la mayor supervivencia de los pacientes alcanzada con nuevos tratamientos como inhibidores del enzima convertidor de angiotensina (IECA) (3), antagonistas del receptor de angiotensina II (ARA-II) (4) y beta-bloqueantes (5). A pesar de ello, los pacientes presentan una calidad de vida incapacitante con múltiples ingresos hospitalarios lo que supone un elevado gasto sanitario (1,8-3,1\% del gasto sanitario global) (6), en España es la primera causa de ingreso hospitalario, alcanzando el 5\% de la totalidad de ingresos, especialmente en mayores de 65 años (7).

Actualmente es incuestionable la relación entre la hipertensión arterial (HTA) y la insuficiencia cardiaca (IC), existiendo una relación lineal entre ambas, en la que a mayores cifras de presión arterial (PA), mayor riesgo de IC (8) y, paralelamente, el tratamiento y control de las cifras de PA se relaciona con la reducción del riesgo de IC $(9,10)$. Disponemos de estudios que demuestran un riesgo atribuible (59 en mujeres y $39 \%$ en hombres) de la HTA para desarrollar IC, siendo muy superior a otros factores como cardiopatía isquémica, diabetes, hipertrofia ventricular izquierda o valvulopatías8. Estos datos se ven reforzados por estudios recientes realizados tanto en servicios de cardiología (11) como de medicina interna (12) que demuestran que la cardiopatía hipertensiva es la primera causa de IC.

A pesar de la evidencia existente a favor del correcto tratamiento en el paciente con insuficiencia cardiaca, se sabe que menos del 5\% de los pacientes cumplen todos los procedimientos diagnósticos y terapéuticos (13).

Por todo lo expuesto previamente se planteó el presente estudio, con el objetivo principal de conocer la prevalencia y grado de control de la hipertensión arterial entre los pacientes con insuficiencia cardiaca.

\section{MATERIAL Y MÉTODOS}

Se realizó un estudio descriptivo transversal retrospectivo en el que se revisó la información contenida en los informes de alta e historia clínica de los pacientes que ingresaron en el Servicio de Medicina Interna del Hospital Clínico Universitario de Santiago de Compostela entre los años 1999 y 2003 (5 años) con diagnóstico clínico de insuficiencia cardíaca al alta, según el cumplimiento de criterios de Framingham (14) (Tabla I).
El registro de datos se realizó retrospectivamente a lo largo de los años 2003 y 2004, registrando las variables indicadas en la tabla II para cada paciente. Se definieron la hipertensión arterial (HTA) y la diabetes mellitus según criterios del Joint Nacional Comité VII (JNC-VII) (9) y de la Asociación Americana de Diabetes (15), respectivamente. Se consideró cardiopatía isquémica a la presencia de síntomas, alteraciones electrocardiográficas (ECG) y/o alteraciones angiográficas compatibles con dicha patología en algún ingreso previo o en el mismo ingreso. Se registró el antecedente de fibrilación auricular (FA) en aquellos casos que presentaran la citada arritmia en el ECG o bien existiera el registro de FA paroxística en la historia del paciente. Se consideró que la fracción de eyección estaba conservada cuando era superior al $45 \%$, deprimida cuando se encontraba entre 30 y $45 \%$ y severamente deprimida cuando era inferior al 30\%. Se recogió como factor desencadenante del episodio el que indicó el facultativo en el informe de alta.

\section{TABLA I}

CRITERIOS FRAMINGHAM PARA EL DIAGNÓSTICO DE INSUFICIENCIA CARDIACA

\begin{tabular}{l} 
Criterios mayores \\
\hline Disnea paroxística nocturna u ortopnea \\
Ingurgitación yugular \\
Estertores \\
Cardiomegalia \\
Edema agudo de pulmón \\
Galope por tercer tono \\
Presión venosa central > $16 \mathrm{mmHg}$ \\
Tiempo de circulación > $25 \mathrm{sg}$ \\
Reflujo hepatoyugular
\end{tabular}

Criterios menores

Edema maleolar

Tos nocturna

Disnea de esfuerzo

Hepatomegalia

Derrame pleural

Reducción de la capacidad vital en un tercio respecto la

máxima

Taquicardia (> 120 lat/min)

Pérdida de $>4,5 \mathrm{Kg}$ en 5 días en respuesta al tratamiento (podría ser criterio mayor)

Para diagnóstico de insuficiencia cardíaca se precisan dos criterios mayores o uno mayor y 2 menores. En los criterios menores deben descartarse otras causas. 


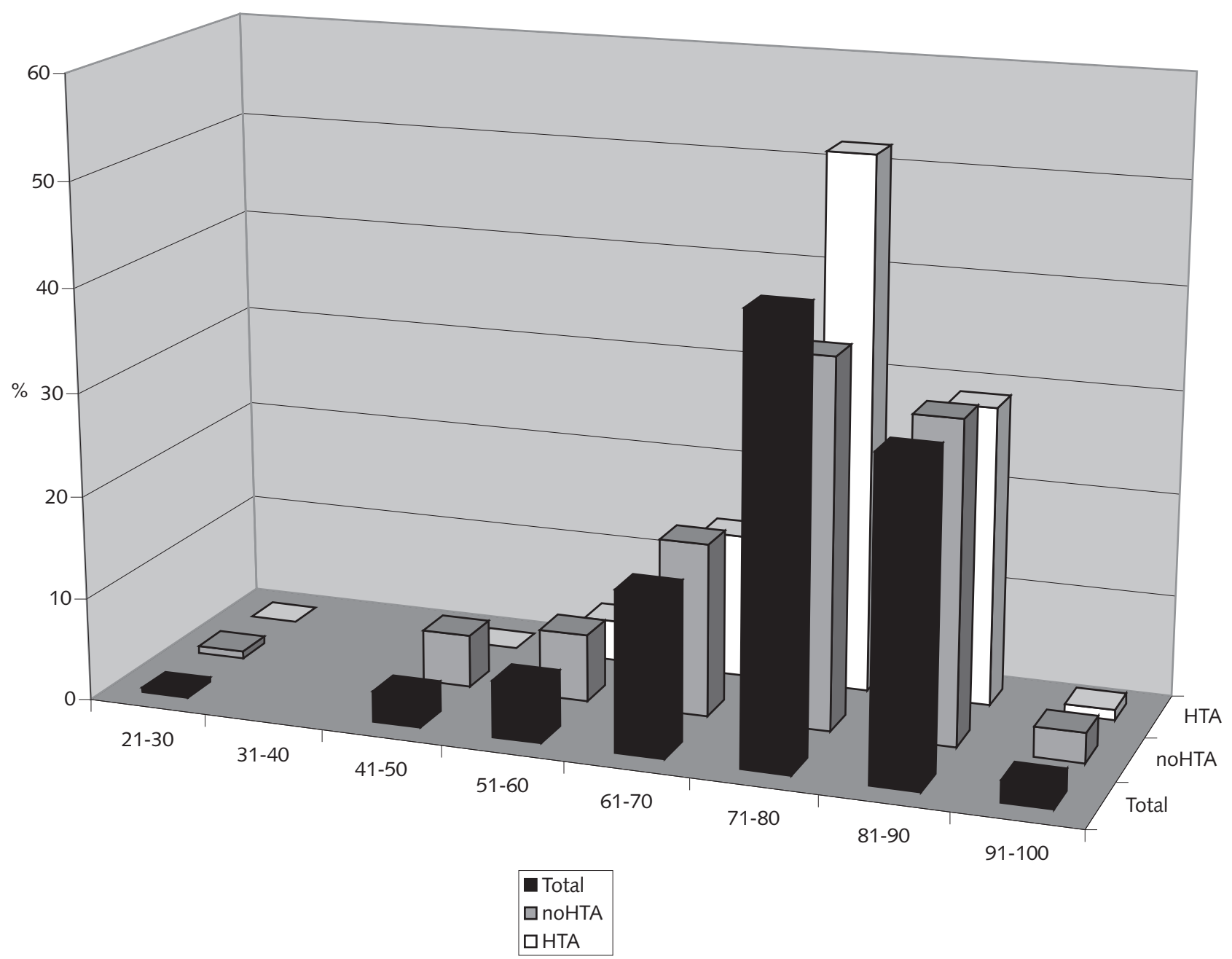

Fig. 1. Distribución por décadas de edad de los pacientes global, y por grupos de hipertensos/no hipertensos.

\section{TABLA II}

\section{VARIABLES REGISTRADAS EN EL ESTUDIO}

\section{Sexo}

Edad

Días de ingreso

Número de reingresos por insuficiencia cardíaca

Motivo de ingreso

Hipertensión arterial (HTA) previa

Diabetes mellitus (DM) previa

Cardiopatía previa

Cardiopatía isquémica previa

Fibrilación auricular (FA) previa

Consumo de beta-bloqueantes previos al ingreso

Cifras de presión arterial (PA) al ingreso

Realización de ecocardiografía durante el ingreso

Fracción de Eyección (FE) según ecocardiograma

Factor desencadenante

Exitus

Tratamiento domiciliario al alta
Para el análisis estadístico se emplearon los estadísticos descriptivos cuantitativos y cualitativos que correspondieran; para el análisis bivariante se emplearon chi-cuadrado y "t-student".

\section{RESULTADOS}

Se incluyeron un total de 248 pacientes con diagnóstico de insuficiencia cardíaca en los 5 años de registro (desde 1999 hasta 2003), de los cuales $100(41,8 \%)$ eran hipertensos. En la población de estudio se observó un predominio de mujeres $(55,2 \%)$, tendencia que se mantuvo en el grupo de hipertensos $(63,0 \%)$, sin que se hallaran diferencias estadísticamente significativas con los no hipertensos $(51,1 \%), \mathrm{p}=0,06$. La mediana de edad fue de 77 años en la población, este mismo datos se corroboró posteriormente entre hipertensos (mediana de 77 años, rango intercuartílico de 10 años) y no hipertensos (mediana de 77 años y rango intercuartílico de 14 años), sin observarse diferencias estadísticamente significativas $(p=0,64)$. La distribución por décadas (Fig. 1) entre ambos grupos también es 


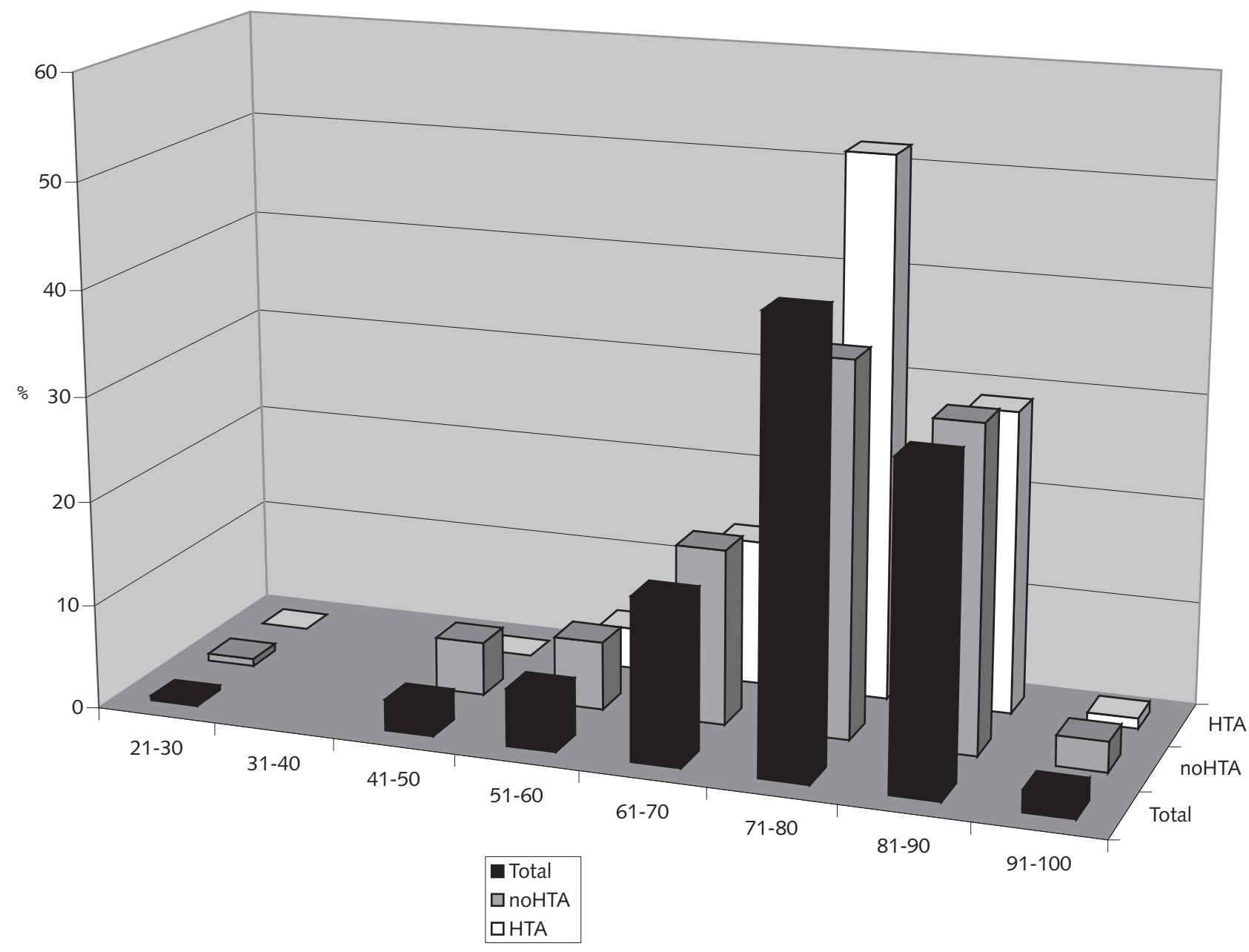

Fig. 2. Motivo de ingreso en la población global, y por grupos de hipertensos y no hipertensos.

similar, siendo predominante el grupo de edad comprendido entre los 71 y 80 años, $p=0,07$.

La mediana de tiempo de ingreso fue de 11 días (rango intercuartílico de 8 días) en la población, cifra que se mantuvo constante entre hipertensos (10 días, rango intercuartílico 8,5 días) y no hipertensos (11 días, rango intercuartílico 9 días).

El análisis de los antecedentes personales mostró que el número de diabéticos era claramente superior en el grupo de hipertensos (43vs. 22,3\%), p < 0,001. En cuanto a la cardiopatía previa, era más frecuente en los hipertensos el presentar algún tipo de patología cardíaca (89 vs. 74,8\%), p = 0,006 ; siendo claramente más frecuente la presencia de cardiopatía isquémica entre los hipertensos (38vs. 21,6\%), $\mathrm{p}=$ 0,005 ; y sin que se observaran diferencias entre la prevalencia de arritmias como la fibrilación auricular (23 vs. 31,6\%), $\mathrm{p}=0,14$.

El síntoma que originó el ingreso con más frecuencia fue la disnea tanto en la población total $(68,9 \%)$ como en hipertensos $(73,0 \%)$ y no hipertensos $(66,9 \%), p=0,62$. En la figura 2 se muestran los síntomas al ingreso en la población total y en cada subgrupo.

En el momento del ingreso el 62,6\% de la población estudiada presentaba cifras anormalmente elevadas de presión arterial (PA). En el caso de los hipertensos, esta prevalencia alcanza el $76,9 \%$, permaneciendo en $59,4 \%$ en no hipertensos, $\mathrm{p}=0,01$. La media de presión arterial sistólica (PAS) en pacientes hipertensos $(146,2 \mathrm{mmHg})$ fue superior a la de pacientes no hipertensos $(130,4 \mathrm{mmHg}), \mathrm{p}<0,01$. La media de presión arterial diastólica (PAD) también es superior en pacientes hipertensos $(83,8 \mathrm{mmHg} v s .76,7 \mathrm{mmHg}), \mathrm{p}<0,01$.

El análisis de los pacientes hipertensos mostró que no había diferencias estadísticamente significativas entre pacientes diabéticos y no diabéticos $(86,7 v s .70,8 \%$, respectivamente), $\mathrm{p}=0,10$. El análisis cuantitativo corrobora este dato, ya que tampoco se observaron diferencias entre diabéticos y no diabéticos en las cifras de PAS $(152,2 \mathrm{mmHg}$ vs. 144,5 $\mathrm{mmHg}$, respectivamente) ni PAD $(83,2 \mathrm{mmHg}$ vs. 84,2 $\mathrm{mmHg}$, respectivamente), $\mathrm{p}=0,16$ y $\mathrm{p}=0,80$, respectivamente. Tampoco se observaron diferencias en el control de las cifras de PA en pacientes hipertensos con o sin cardiopatía isquémica previa ( $82,1 v s .74,0 \%$, respectivamente), $\mathrm{p}=0,41$. Las cifras medias de PAS (142 mmHg vs. $148 \mathrm{mmHg}$, respectivamente) y PAD $(85,1 \mathrm{mmHg}$ vs. $83,0 \mathrm{mmHg}$ respectivamente) también fueron similares entre ambos grupos de cardiópatas, $\mathrm{p}=0,39$ y $\mathrm{p}=0,60$, respectivamente.

El número de pacientes diabéticos descompensados es similar entre hipertensos y no hipertensos $(52,2$ vs. $60,5 \%)$, p $=0,23$, en cambio las cifras de glucemia fueron significativa- 


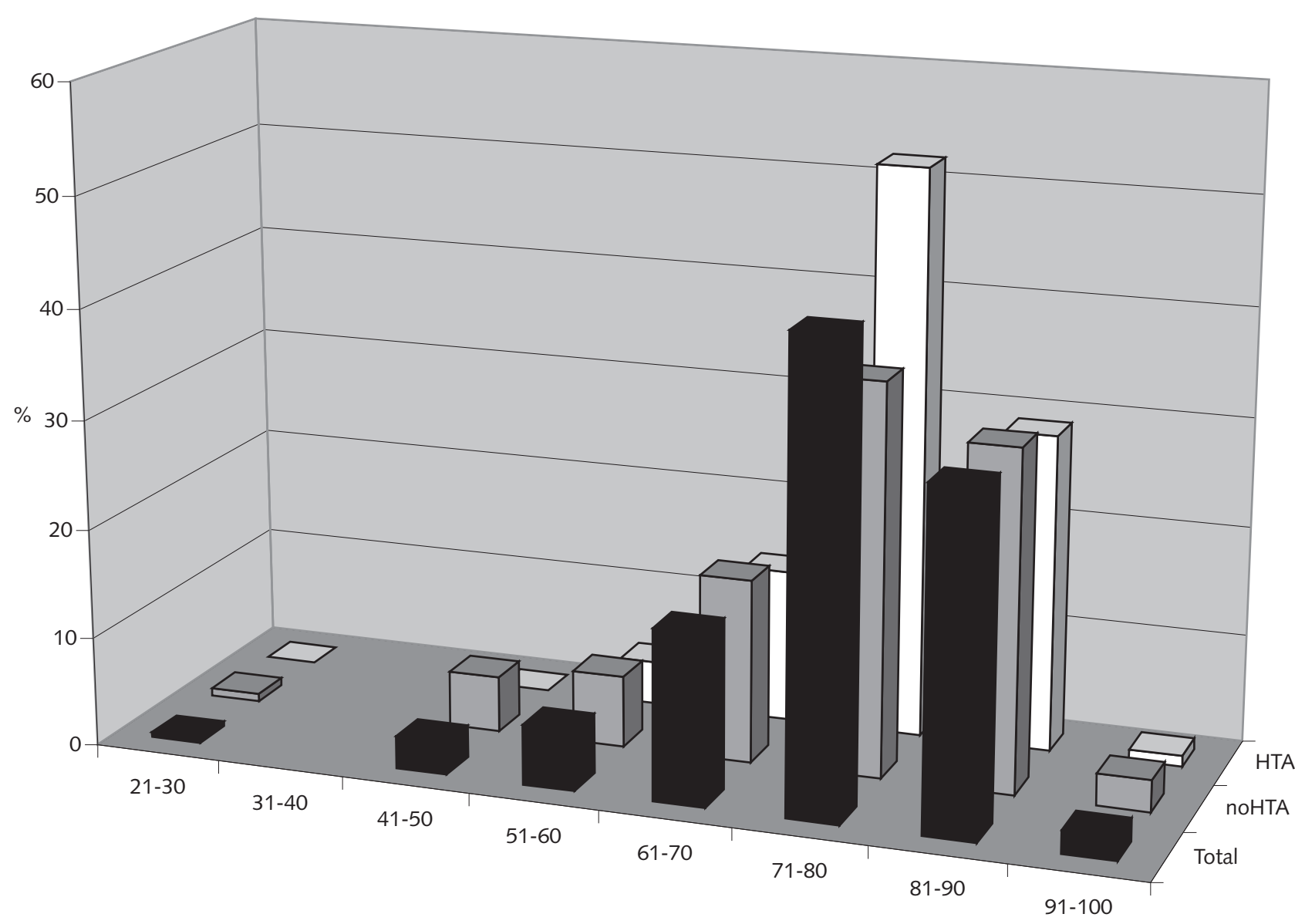

Fig. 3. Distribución de los factores de descompensación en la población global, y por grupos de hipertensos y no hipertensos.

mente superiores en los pacientes hipertensos $(173,8 \mathrm{mg} / \mathrm{dl} v s$. $145,1 \mathrm{mg} / \mathrm{dl}), \mathrm{p}=0,04$.

Se realizó ecocardiograma al 21,3\% de los pacientes estudiados. No se observaron diferencias en el análisis de pacientes hipertensos y no hipertensos (16,3 vs. 24,8\%), $\mathrm{p}=0,12$. Entre estos pacientes a los que se les realizó ecocardiograma, el porcentaje de pacientes con función sistólica conservada fue ligeramente superior en el grupo de hipertensos $(62,5 \mathrm{vs}$. $50,5 \%)$, sin que se hallaran diferencias estadísticamente significativas $(\mathrm{p}=0,41)$.

Entre las causas de descompensación cardíaca, la distribución entre hipertensos es similar a la mostrada en la Parte I del estudio de la población global (Fig. 3): causa desconocida $(34,0 \%)$, infección respiratoria $(39,0 \%)$, arritmias $(7,0 \%)$, anemia $(7,0 \%)$ y valvulopatías $(4,0 \%)$. Tampoco se observaron diferencias entre hipertensos y normotensos en estas distribuciones, $\mathrm{p}=0,67$.

El estudio de pacientes fallecidos tampoco mostró diferencias con el de la población global $(8,6 \%)$. Entre los pacientes hipertensos, el porcentaje de fallecidos alcanzó el 6,1\% frente al $10,1 \%$ entre pacientes no hipertensos, esta diferencia no fue estadísticamente significativa $(\mathrm{p}=0,27)$. Igualmente se obser- vó en el grupo de hipertensos, mayor edad media entre los pacientes fallecidos (81 vs. 76 años, $\mathrm{p}=0,05)$.

Entre los tratamientos administrados se observó que se administraban más diuréticos en el grupo de pacientes no hipertensos $(91,1 v s .81,1 \%, p=0,03)$. El grupo IECA/ARA-II fue significativamente más administrado en el grupo de hipertensos $(62,6$ vs. 26,8\%,p < 0,001). Los calcio-antagonistas se administraron mayoritariamente en el grupo de pacientes hipertensos, que recibieron el $81 \%$ de los prescritos, $(9,9$ vs. $1,6 \%, \mathrm{p}=$ $0,006)$. La administración de nitratos transdérmicos $(54,9$ vs. $46,3 \%)$ y orales $(5,5$ y $10,6 \%)$ fue similar en ambos grupos, $\mathrm{p}=$ y $\mathrm{p}=0,18$, respectivamente. Entre los dos beta-bloqueantes administrados, se repartieron uno para cada grupo.

\section{DISCUSIÓN}

Las características epidemiológicas de nuestros grupos de estudio coinciden con otros estudios realizados sobre otras poblaciones de mayor tamaño, como son el predominio de sexo femenino, que además presenta mayor mediana de edad y la cardiopatía isquémica e hipertensiva como las dos prime- 
TABLA III

FÁRMACOS EMPLEADOS EN EL TRATAMIENTO DE LA INSUFICIENCIA CARDIACA EN LA POBLACIÓN DE ESTUDIO, Y POR HIPERTENSOS/NO HIPERTENSOS

\begin{tabular}{lccc}
\hline & Global & HTA & No HTA \\
\hline Diuréticos & $186(86,9 \%)$ & $74(81,3 \%)$ & $112(91,1 \%)$ \\
Nitratos transdérmicos & $107(50,0 \%)$ & $50(54,9 \%)$ & $57(46,3 \%)$ \\
IECA / ARA-II & $90(42,1 \%)$ & $57(62,6 \%)$ & $33(26,8 \%)$ \\
Nitratos orales & $18(8,4 \%)$ & $5(5,5 \%)$ & $13(10,6 \%)$ \\
Antagonistas del calcio & $11(5,1 \%)$ & $9(9,9 \%)$ & $2(1,6 \%)$ \\
Vasodilatadores arteriales & $15(7,1 \%)$ & $12(13,2 \%)$ & $3(2,4 \%)$ \\
Beta-bloqueantes & $2(0,9 \%)$ & $1(1,1 \%)$ & $1(0,8 \%)$ \\
\hline
\end{tabular}

ras causas de insuficiencia cardiaca $(16,17)$. Por otro lado, la mediana de edad de nuestro grupo (71 años) corresponde a la aportada por otros estudios internaciones (tanto europeos (18) como americanos) (19) como nacionales, por ejemplo el Estudio CARDIOTENS (20) realizado con población correspondiente a consultas de Cardiología y Atención Primaria.

Teniendo como punto de partida una población similar a la de otros centros de características similares, observamos que el tiempo de estancia media de los pacientes con insuficiencia cardíaca es de 11 días, cifra que se coincide con los datos aportados por estudios nacionales (21) e internacionales (22).

A pesar de tratarse de dos patologías muy prevalentes, no se dispone en España, de datos de prevalencia de HTA en IC $(23,24)$. El presente estudio aporta este dato $(41,8 \%)$, que sin pretender ser definitivo, sí abre una puerta en este camino. Esta prevalencia es ligeramente superior a la observada en la población general, entorno al 30\% (25).

En el análisis epidemiológico (Parte I) ya se realizó el comentario correspondiente al reducido número de ecocardiogramas realizado a los pacientes del Servicio de Medicina Interna. Dicho comentario es extrapolable a los pacientes hipertensos estudiados en el actual documento9,26. Como llamada de atención, es importante recordar dos cuestiones: la disfunción diastólica es difícil de valorar con datos exclusivamente clínicos (sin ecocardiograma) y, además la prevalencia de disfunción diastólica entre pacientes HTA alcanza e incluso podría superar el 40\%19; por lo que se hace inexcusable la realización de un ecocardiograma a todo paciente con insuficiencia cardiaca e hipertensión para poder realizar un adecuado abordaje terapéutico del mismo.

El número de antihipertensivos prescritos muestra cierta tendencia a emplear IECA/ARA-II en pacientes hipertensos, posiblemente por la elevada asociación con diabetes mellitus en nuestra población. En contraste con lo presentado en el grupo de población global, el empleo de fármacos como los IECAs/ARA-II en este grupo de hipertensos, que presentan además insuficiencia cardiaca, $(62,6$ y $1 \%$, respectivamente), se aproxima al de los grandes registros como el estudio BADAPIC16 (87\%) o el Euro.Heart-Failure Survey (17) (66\%); en cambio, los beta-bloqueantes prescritos son muy escasos (1\% de los pacientes hipertensos), frente al 59\% y $37 \%$ mostrado por estos dos estudios.

Es obvio que existen diferencias en el tratamiento de los pacientes con insuficiencia e hipertensión por parte de médicos especialistas en Medicina Interna y Cardiólogos, como así lo demuestran innumerables estudios27,28. Estas diferencias probablemente se deban fundamentalmente a cuestiones de pluripatología, como son las características clínicas entre los pacientes ingresados en Medicina Interna (EPOC, asma, vasculopatía periférica, diabetes mellitus, etc) y la escasa accesibilidad en la realización de ecocardiogramas en un tiempo aceptablemente corto, que evite demorar el alta del paciente de forma innecesaria, y que permita una más correcta prescripción terapéutica.

Entre las limitaciones del estudio destacan el diseño descriptivo observacional que no permite establecer relaciones causales, y el reducido tamaño muestral, que ha dificultado en varias ocasiones identificar diferencias estadísticamente significativas. Ambas limitaciones favorecen un sesgo de selección, sin embargo, creemos han sido superadas al seleccionar toda la población de pacientes con insuficiencia cardiaca ingresada a lo largo de 5 años, por lo que en realidad no se trata de una muestra sino de una población. Esta idea se ve reforzada por el hecho de que los datos epidemiológicos de nuestra población coinciden exactamente con los datos aportados por estudio y registros con un tamaño muestral muy elevado (16-20).

Tras lo expuesto podemos concluir que: existe una elevada asociación de pacientes hipertensos y diabéticos, lo que obliga a realizar determinados ajustes terapéuticos en el paciente con insuficiencia cardíaca. La realización de ecocardiogramas es muy baja en todos los pacientes, particularmente en hipertensos. El empleo de IECAs es aceptable, aproximándose al de registros internacionales. La pluripatología de los pacientes de un servicio de Medicina Interna y la escasez de ecocardiogramas realizados pueden estar relacionados con la mínima administración de beta-bloqueantes.

Sería necesario realizar un estudio longitudinal que permita identificar los factores asociados a los problemas que se han identificado, además de poder identificar las fórmulas más adecuadas para su corrección.

\section{Bibliografía}

1. Cortina A, Reguero J, Segovia E, Rodríguez Lambert JL, Cortina R, Arias JC, et al. Prevalence of heart failure in Asturias (a region in the North of Spain). Am J Cardiol 2001; 87: 1417-9.

2. Cleland JGF, Khand A, Clark A. The heart failure epidemic: exactly how big is it? Eur Heart J 2001; 22: 623-6.

3. Cleland JGF, Gemmel I, Khand A, Boddy A. Is the prognosis of heart failure improving? Eur J Heart Fail 1999; 1: 229-41.

4. Barrios V, Ortega J. ACE inhibitors in the treatment of heart failure. Heart Failure Train Lett 1999; 7: 2-5.

5. Poole-Wilson PA, Swedberg K, Cleland JGF, DiLenarda A, Hanrath P,

Komadja M, et al, for the COMET Investigators. Comparison of carvedilol and metoprolol on clinical outcomes in patients with chronic heart failure in the Carvedilol or Metoprolol European Trial (COMET): Randomised controlled trial. Lancet 2003: 362: 7-13.

6. Antoñanzas F. Costes de la insuficiencia cardíaca en España. An Med Interna (Madrid) 1997; 14: 9.

7. Rodríguez-Artalejo F, Banegas Banegas JR, Guayar-Castillón P. Epidemiología de la insuficiencia cardiaca. Rev Esp Cardiol 2004; 57: 163-70.

8. Levy D, Larson MG, Vasan RS, Kannel WB, Ho KKL. The progression from hypertension to congestive heart failure. JAMA 1996; 275: 1557-62 
9. Joint National Committee on Prevention, Detection, Evaluation, and Treatment og High Blood Pressure. The Seventh report of the Joint National Committee on Prevention, Detection, Evaluation, and Treatment of High Blood Presure (JNC VII). JAMA 2003; 289: 2560-72.

10. Lombera F, Barrios V, Soria F, Placer L, Cruz JM, Tornás L, et al. Guías de práctica clínica de la Sociedad Española de Cardiología en hipertensión arterial. Rev Esp Cardiol 2000; 53: 66-90.

11. González Juanatey JR, Alegría E, Vidal JV, Caro JL, García Acuña M, et al. Impacto de la hipertensión sobre las enfermedades cardíacas en España. El estudio CARDIOTENS 1999. Rev Esp Cardiol. 2001; 54: 139-49.

12. Grupo de Trabajo de Insuficiencia Cardíaca de la Sociedad Española de Medicina Interna (SEMI). Insuficiencia cardíaca en lso departamentos de Medicina Interna. Med Clin (Barc) 2002; 118: 605-10.

13. McCullough PA, Philbin EF, Spertus JA, Sandberg KR, Sullivan RA, Kaatz S. Opportunities for improvement in the diagnosis and treatment on heart failure. Cin Cardiol. 2003; 26 (5): 231-7.

14. Braunwald E. The pathogenesis of the heart failure: then and now. Medicine 1991; 70: 68.

15. Expert Committee on the diagnosis and classification of Diabetes Mellitus. American Diabetes Association: clinical practice recommendations 2002. Diabetes Care 2002 Jan; 25 Suppl 1: S1-147.

16. Anguita Sánchez M, et al. Características clínicas, tratameinto y morbimortalidad a corto plazo de pacientes con insuficiencia cardíaca controlados en consultas específicas de insuficiencia cardiaca. Resultados del Registro Badapic. Rev Esp Cardiol 2004; 57: 1159-69

17. Komadja M, Follath F, Swedberg K, Cleland J, Aguilar JC, CohenSolal A, et al. The Euroheart Failure Survey programme. A survey on the quality of care among patients with heart failure in Europe part 2: treatment. Eur Heart J 2003; 24: 464-74

18. Cowle MR, Word DA, Cotas AJS, Thomson SG, Poole-Wilson PA, Suresh V, et al. Incidence and aetiology of heart failure: A populationbased study. Eur Heart J 1999; 20: 421-8.

19. Senni M, Tribouilloy CM, Rodeheffer RJ, JAcobsen SJ, Evans JM, Bai- ley KR, et al. Congestive Herat failure in the comunity. A study of all incident cases in Olmsted Country. Minnesonta in 1991. Circulation 1998; 98: 2282-9.

20. Barrios Alonso et al. Hipertensión Arterial e Insuficiencia Cardiaca en las consultas de Atención Primaria y Cardiología de España. Rev Clin Esp 2003; 203: 334-42.

21. García Castelo A, Muñiz García J, Sesma Sánchez P, Castro Beiras A, en representación del grupo de estudio INCARGAL. Utilización de recursos diagnósticos y terapéuticos en pacientes ingresados por insuficiencia cardíaca: influencia del servicio de ingreso (estudio INCARGAL). Rev Esp Cardiol 2003; 56: 49-56.

22. Cleland JGF, Swedberg K, Folath F, Komjda M, Cohen-Solal A, Aguilar JC, et al. The EuroHeart Failure survey programme a survey on the quality of care among patients with heart failure in Europe. Part 1: patient characteristics and diagnosis. Eur Heart J 2003; 24: 442-63.

23. Brotons C, Moral I, Ribera A, Pérez G, Cascant P, Bustins M, et al. Tendencias de la morbimortalidad por insuficiencia cardíaca en Cataluña. Rev Esp Cardiol 1998; 51: 972-6.

24. Rodríguez Artalejo F, Guallar P, Banegas JR, Del Rey J. Trends in hospitalization and mortality for Herat failure in Spain 1980-1993. Eur Heart J 1997; 18: 1771-9.

25. Albaladejo Blanco C, Martín García JA, López López I, Ródenas Aguilar JL, Ingla Mas J, López Vivo J. Grupos de riesgo cardiovascular y adecuación del tratamiento farmacológico en hipertensos de edad avanzada. Hipertensión 2001; 18: 305-13.

26. ACC/AHA Guidelines for the evaluation and treatment of chronic heart failure. European Society of Cardiology. Eur Heart J 2001; 22: 1527-60.

27. Bellotti L, Badano LP, Acquarine N, Griffo R, Lo Pinto G, Maggioni A et al. Speciality-related differences in the epidemiology, clinical profile, management and outcome of patients hospitalized for heart failure. The OSCUR Study. Eur Heart J 2001; 22: 596-604.

28. Bonarjee VVS, Dickstein K. Management of patients with heart failure: are internists as good as cardiologists? Eur Heart J 2001; 22: 530-1. 